International Journal of Mathematical Analysis

Vol. 9, 2015, no. 40, 1955 - 1970

HIKARI Ltd, www.m-hikari.com

http://dx.doi.org/10.12988/ijma.2015.57176

\title{
A Method to Solve Non-homogeneous Strongly Coupled Mixed Parabolic Boundary Value Systems with Non-homogeneous Boundary Conditions
}

\author{
Vicente Soler \\ Departamento de Matemática Aplicada \\ Universitat Politècnica de València, Spain \\ Emilio Defez \\ Instituto de Matemática Multidisciplinar \\ Universitat Politècnica de València, Spain \\ Roberto Capilla \\ Departamento de Ingeniería Electrónica \\ Universitat Politècnica de València, Spain
}

Copyright (c) 2015 Vicente Soler, Emilio Defez and Roberto Capilla. This article is distributed under the Creative Commons Attribution License, which permits unrestricted use, distribution, and reproduction in any medium, provided the original work is properly cited.

\begin{abstract}
In this paper, a method to construct the solution of non-homogeneous parabolic coupled systems with non-homogeneous boundary conditions of the type $u_{t}-A u_{x x}=G(x, t), A_{1} u(0, t)+B_{1} u_{x}(0, t)=P(t), A_{2} u(l, t)+$ $B_{2} u_{x}(l, t)=Q(t), 0<x<1, t>0, u(x, 0)=f(x)$, where $A$ is a positive stable matrix and $A_{1}, A_{2}, B_{1}, B_{2}$ are arbitrary matrices for which the block matrix $\left(\begin{array}{cc}A_{1} & B_{1} \\ A_{2} & B_{2}\end{array}\right)$ is non-singular, is proposed. Two illustrative examples of the method are given.
\end{abstract}




\section{Mathematics Subject Classification: 35K50}

Keywords: Coupled diffusion problems, coupled boundary conditions, vector boundary-value differential systems, non-homogeneous problems, nonhomogeneous conditions.

\section{Introduction}

Coupled partial differential systems with coupled boundary-value conditions are frequent in different areas of science and technology. Recently, an exact series solution for the homogeneous initial-value problem

$$
\begin{aligned}
u_{t}(x, t)-A u_{x x}(x, t) & =0,0<x<1, t>0 \\
A_{1} u(0, t)+B_{1} u_{x}(0, t) & =0, t>0 \\
A_{2} u(1, t)+B_{2} u_{x}(1, t) & =0, t>0 \\
u(x, 0) & =f(x), 0 \leq x \leq 1
\end{aligned}
$$

where $u=\left(u_{1}, u_{2}, \ldots, u_{m}\right)^{T}$ and $f(x)=\left(f_{1}(x), f_{2}(x), \ldots, f_{m}(x)\right)^{T}$ are a $m$-dimensional vectors, was constructed under the following hypotheses and notation:

1. The matrix coefficient $A$ is a matrix which satisfies the following condition

$$
\operatorname{Re}(z)>0, \forall z \in \sigma(A)
$$

where $\sigma(C)$ denotes the set of all the eigenvalues of a matrix $C$ in $\mathbb{C}^{m \times m}$. Thus $A$ is a positive stable matrix (where $\operatorname{Re}(z)$ denotes the real part of $z \in \mathbb{C})$.

2. Matrices $A_{i}, B_{i}, i=1,2$, are $m \times m$ complex matrices, and we assume that the block matrix

$$
\left(\begin{array}{ll}
A_{1} & B_{1} \\
A_{2} & B_{2}
\end{array}\right) \text { is regular }
$$

and also that the matrix pencil

$$
A_{1}+\rho B_{1} \text { is regular. }
$$

Condition (7) is well known in the literature of singular systems of differential equations, see [1], and involves the existence of some $\rho_{0} \in \mathbb{C}$ so that matrix $A_{1}+\rho_{0} B_{1}$ is invertible. In this case, matrix $A_{1}+\rho B_{1}$ is invertible with the possible exception of at most a finite number of complex numbers $\rho$. In particular, we may assume that $\rho_{0} \in \mathbb{R}$. 
Using condition (7) we can introduce the following matrices $\widetilde{A}_{1}$ and $\widetilde{B}_{1}$ defined by

$$
\widetilde{A}_{1}=\left(A_{1}+\rho_{0} B_{1}\right)^{-1} A_{1}, \widetilde{B}_{1}=\left(A_{1}+\rho_{0} B_{1}\right)^{-1} B_{1}
$$

which satisfy the condition $\widetilde{A}_{1}+\rho_{0} \widetilde{B}_{1}=I$, where matrix $I$ denotes, as usual, the identity matrix. Under hypothesis (6), is it easy to show that matrix $B_{2}-\left(A_{2}+\rho_{0} B_{2}\right) \widetilde{B}_{1}$ is regular and we can introduce matrices $\widetilde{A}_{2}$ and $\widetilde{B}_{2}$ defined by

$$
\widetilde{A}_{2}=\left[B_{2}-\left(A_{2}+\rho_{0} B_{2}\right) \widetilde{B}_{1}\right]^{-1} A_{2}, \widetilde{B}_{2}=\left[B_{2}-\left(A_{2}+\rho_{0} B_{2}\right) \widetilde{B}_{1}\right]^{-1} B_{2}
$$

that satisfy the conditions $\widetilde{B}_{2}-\left(\widetilde{A}_{2}+\rho_{0} \widetilde{B}_{2}\right) \widetilde{B}_{1}=I, \widetilde{B}_{2} \widetilde{A}_{1}-\widetilde{A}_{2} \widetilde{B}_{1}=I$.

Under the above assumptions, the homogeneous problem (1)-(4) was solved in $[2,3]$ under two different cases:

(a) We can consider the following hypothesis:

$$
\begin{gathered}
\text { exist } b_{1} \in \sigma\left(\widetilde{B}_{1}\right)-\{0\}, b_{2} \in \sigma\left(\widetilde{B}_{2}\right), \text { and } v \in \mathbb{C}^{m}-\{0\} \\
\text { such that }\left(\widetilde{B}_{1}-b_{1} I\right) v=\left(\widetilde{B}_{2}-b_{2} I\right) v=0
\end{gathered}
$$

Then, if the vector valued function $f(x)$ satisfies hypotheses

$$
\left.\begin{array}{c}
f \in \mathcal{C}^{2}([0,1]) \\
\left(1-\rho_{0} b_{1}\right) f(0)+b_{1} f^{\prime}(0)=0 \\
-\left(\frac{1-b_{2}+\rho_{0} b_{1} b_{2}}{b_{1}}\right) f(1)+b_{2} f^{\prime}(1)=0
\end{array}\right\},
$$

with the additional condition:

$$
f(x) \in \operatorname{Ker}\left(\widetilde{B}_{1}-b_{1} I\right) \cap \underset{\text { and }}{\operatorname{Ker}}\left(\widetilde{B}_{2}-b_{2} I\right), 0 \leq x \leq 1
$$

$\operatorname{Ker}\left(\widetilde{B}_{1}-b_{1} I\right) \cap \operatorname{Ker}\left(\widetilde{B}_{2}-b_{2} I\right)$ is an invariant subspace with respect to matrix $A$,

where a subspace $E$ of $\mathbb{C}^{m}$ is invariant by the matrix $A \in \mathbb{C}^{m \times m}$, if $A(E) \subset E$, we can construct an exact series solution $u(x, t)$ of homogeneous problem (1)-(4). This construction was made in Ref. [2]. 
(b) We can consider the following hypothesis:

$$
\begin{gathered}
0 \in \sigma\left(\widetilde{B}_{1}\right), a_{2} \in \sigma\left(\widetilde{A}_{2}\right) \text {, and we have } w \in \mathbb{C}^{m}-\{0\} \\
\text { so that } \widetilde{B}_{1} w=\left(\widetilde{A}_{2}-a_{2} I\right) w=0
\end{gathered}
$$

Then, if the vector valued function $f(x)$ satisfies the hypotheses

$$
\left.\begin{array}{c}
f \in \mathcal{C}^{2}([0,1]) \\
f(0)=0 \\
a_{2} f(1)+f^{\prime}(1)=0
\end{array}\right\},
$$

under the additional condition:

$$
f(x) \in \operatorname{Ker}\left(\widetilde{B}_{1}\right) \cap \operatorname{Ker}\left(\widetilde{A}_{2}-a_{2} I\right), 0 \leq x \leq 1
$$

$\operatorname{Ker}\left(\widetilde{B}_{1}\right) \cap \operatorname{Ker}\left(\widetilde{A}_{2}-a_{2} I\right)$ is an invariant subspace respect to matrix $A$,

then we can construct an exact series solution $u(x, t)$ of homogeneous problem (1)-(4). This construction was made in Ref. [3].

By other hand, the solution of the non-homogeneous problem

$$
\begin{aligned}
u_{t}(x, t)-A u_{x x}(x, t) & =G(x, t), 0<x<1, t>0 \\
A_{1} u(0, t)+B_{1} u_{x}(0, t) & =0, t>0 \\
A_{2} u(1, t)+B_{2} u_{x}(1, t) & =0, t>0 \\
u(x, 0) & =f(x), 0 \leq x \leq 1
\end{aligned}
$$

was made in Ref. [4] under the two different hypotheses $(a)$ and $(b)$.

This paper deals a method to construct the exact solution of the nonhomogeneous problem with non-homogeneous conditions

$$
\begin{aligned}
u_{t}(x, t)-A u_{x x}(x, t) & =G(x, t), 0<x<1, t>0 \\
A_{1} u(0, t)+B_{1} u_{x}(0, t) & =P(t), t>0 \\
A_{2} u(1, t)+B_{2} u_{x}(1, t) & =Q(t), t>0 \\
u(x, 0) & =f(x), 0 \leq x \leq 1,
\end{aligned}
$$


in term of solutions of problems of the type (16)-(19). Throughout this paper we will assume the results and nomenclature given in $[2,3,4]$. This paper is organized as follows: In section 2 a method to construct a solution of (16)-(19) is obtained. In section 3 an algorithm and two illustrative examples are given. Conclusion are presented in section 4 .

\section{The proposed method}

We consider the non-homogeneous problem with non-homogeneous conditions

$$
\begin{aligned}
u_{t}(x, t)-A u_{x x}(x, t) & =G(x, t), 0<x<1, t>0 \\
A_{1} u(0, t)+B_{1} u_{x}(0, t) & =P(t), t>0 \\
A_{2} u(1, t)+B_{2} u_{x}(1, t) & =Q(t), t>0 \\
u(x, 0) & =f(x), 0 \leq x \leq 1
\end{aligned}
$$

where $u(x, t), G(x, t), P(t), Q(t)$ and $f(x)$ are vectors in $\mathbb{C}^{m}$, and matrices $A_{1}, A_{2}, B_{1}, B_{2} \in \mathbb{C}^{m \times m}$ satisfying the conditions (5) and (6)-(7).

We are looking for a solution of (20)-(23) in the form

$$
u(x, t)=w(x, t)+v(x, t),
$$

where function $v(x, t)$ satisfies the conditions

$$
\left.\begin{array}{l}
A_{1} v(0, t)+B_{1} v_{x}(0, t)=P(t), t>0, \\
A_{2} v(1, t)+B_{2} v_{x}(1, t)=Q(t), t>0 .
\end{array}\right\} .
$$

Thus, we can define the function $G_{1}(x, t)$ as

$$
G_{1}(x, t)=v_{t}(x, t)-A v_{x x}(x, t)
$$

then $v(x, t)$ satisfies:

$$
\begin{aligned}
v_{t}(x, t)-A v_{x x}(x, t) & =G_{1}(x, t), 0<x<1, t>0 \\
A_{1} v(0, t)+B_{1} v_{x}(0, t) & =P(t), t>0 \\
A_{2} v(1, t)+B_{2} v_{x}(1, t) & =Q(t), t>0
\end{aligned}
$$

which implies that $w(x, t)$ must satisfy 


$$
\begin{aligned}
w_{t}(x, t)-A w_{x x}(x, t) & =G(x, t)-G_{1}(x, t) \\
& =\widetilde{G}(x, t), 0<x<1, t>0
\end{aligned}
$$

with the homogeneous conditions:

$$
\left.\begin{array}{l}
A_{1} w(0, t)+B_{1} w_{x}(0, t)=0, t>0, \\
A_{2} w(1, t)+B_{2} w_{x}(1, t)=0, t>0 .
\end{array}\right\} .
$$

and the initial condition:

$$
\begin{aligned}
w(x, 0) & =f(x)-v(x, 0) \\
& =\widetilde{f}(x), 0 \leq x \leq 1 .
\end{aligned}
$$

Then, function $u(x, t)$ defined by (24) satisfy:

$$
\begin{aligned}
u_{t}(x, t)-A u_{x x}(x, t) & =v_{t}(x, t)-A v_{x x}(x, t)+w_{t}(x, t)-A w_{x x}(x, t) \\
& =G_{1}(x, t)+G(x, t)-G_{1}(x, t) \\
& =G(x, t)
\end{aligned}
$$

with the boundary conditions (21)-(22) and the initial condition (23), so it is the desired solution of our problem (20)-(23).

Summarizing, the following theorem has been proved:

Theorem 2.1 Let be consider the problem (20)-(23). Let $v(x, t)$ be a vector valued function satisfying conditions (25). We define the vector valued functions

$$
\widetilde{G}(x, t)=G(x, t)-G_{1}(x, t), \widetilde{f(x)}=f(x)-v(x, 0),
$$

where $G_{1}(x, t)$ is given by (26). We consider the non-homogeneous problem with homogeneous conditions

$$
\begin{aligned}
w_{t}(x, t)-A w_{x x}(x, t) & =\widetilde{G}(x, t), 0<x<1, t>0 \\
A_{1} w(0, t)+B_{1} w_{x}(0, t) & =0, t>0 \\
A_{2} w(1, t)+B_{2} w_{x}(1, t) & =0, t>0 \\
w(x, 0) & =\widetilde{f(x)}, 0 \leq x \leq 1,
\end{aligned}
$$

which solution $w(x, t)$ can be obtain using Theorem 2.1 of Ref. [4] if conditions established in this theorem holds. Then, $u(x, t)=v(x, t)+w(x, t)$ is a solution of problem (20)-(23). 


\section{Algorithm and Examples}

We can establish the following algorithm to solve problem (20)-(23):

Algorithm 1 Solution of problem (20)-(23).

Input data: Matrices $A, A_{1}, A_{2}, B_{1}, B_{2} \in \mathbb{C}^{m \times m}$, vectors $G(x), f(x) \in \mathbb{C}^{m}$.

Result obtained: If the stated assumptions are met, the series solution $u(x, t)$.

1: Determine a vector valued function $v(x, t)$ satisfying conditions (25).

2: Determine $\widetilde{G}(x, t)=G(x, t)-G_{1}(x, t)$ and $\widetilde{f(x)}=f(x)-v(x, 0)$.

3: Using the Algorithm given in Ref. [4] determine, if it is possible, a solution $w(x, t)$ of problem $(27)-(30)$.

4: Determine the solution of problem $(20)-(23)$ as $u(x, t)=w(x, t)+v(x, t)$.

Of course, the choice of the function $v(x, t)$ determine the choice of the functions $\widetilde{G}(x, t)$ and $\widetilde{f}(x)$, which must satisfy the hypotheses of Theorem 2.1 of Ref. [4], and depend on the nature of the given function $G(x, t)$. Here we present two different examples.

Example 3.1 We consider problem (20)-(23) where function $G(x, t)$ is a linear combination of functions $\sin (\pi x)$ and $\cos (\pi x)$. Then, we will look for a function $v(x, t)$ which is also a linear combination of functions $\sin (\pi x)$ and $\cos (\pi x)$ with coefficients are functions of variable $t$. Thus, we look for a solution of (25) given in the form

$$
v(x, t)=R_{1}(t) \sin (\pi x)+R_{2}(t) \cos (\pi x),
$$

where vector-valued functions $R_{i}(t) \in \mathcal{C}^{1}[0,+\infty), i=1,2$ must be determinate. This solution (31) must to satisfy boundary conditions (25):

$$
\left.\begin{array}{l}
A_{1} v(0, t)+B_{1} v_{x}(0, t)=P(t) \Longrightarrow A_{1} R_{2}(t)+\pi B_{1} R_{1}(t)=P(t) \\
A_{2} v(1, t)+B_{2} v_{x}(1, t)=Q(t) \Longrightarrow-A_{2} R_{2}(t)-\pi B_{2} R_{1}(t)=Q(t)
\end{array}\right\}
$$

Writing (32) in matrix form:

$$
\left(\begin{array}{rr}
A_{1} & B_{1} \\
-A_{2} & -B_{2}
\end{array}\right)\left(\begin{array}{c}
R_{2}(t) \\
\pi R_{1}(t)
\end{array}\right)=\left(\begin{array}{c}
P(t) \\
Q(t)
\end{array}\right) .
$$

Premultiplying (33) by the invertible matrix $\left(\begin{array}{rr}I & 0 \\ 0 & -I\end{array}\right)$ one gets

$$
\left(\begin{array}{cc}
A_{1} & B_{1} \\
A_{2} & B_{2}
\end{array}\right)\left(\begin{array}{c}
R_{2}(t) \\
\pi R_{1}(t)
\end{array}\right)=\left(\begin{array}{c}
P(t) \\
-Q(t)
\end{array}\right) .
$$


Under hypothesis (6) this system has an unique solution. Thus, we have shown that we can determine a vector valued function $v(x, t)$ satisfying conditions (25) and defined by expression (31). Thus, we have now that

$$
\begin{aligned}
G_{1}(x, t) & =v_{t}(x, t)-A v_{x x}(x, t) \\
& =R_{1}^{\prime}(t) \sin (\pi x)+R_{2}^{\prime}(t) \cos (\pi x)+\pi^{2} A v(x, t),
\end{aligned}
$$

and we can apply Theorem 2.1. We will consider a concrete numerical example. Consider problem (20)-(23) where matrix $A \in \mathbb{C}^{4 \times 4}$ is given by

$$
A=\left(\begin{array}{rrrr}
2 & 0 & 0 & 1 \\
1 & 2 & 0 & -2 \\
-1 & 0 & 2 & 1 \\
0 & 0 & 0 & 1
\end{array}\right)
$$

and the $4 \times 4$ matrices $A_{i}, B_{i}, i \in\{1,2\}$, are

$$
\begin{array}{ll}
A_{1}=\left(\begin{array}{llll}
0 & 0 & 0 & 0 \\
0 & 0 & 0 & 0 \\
0 & 0 & 1 & 0 \\
0 & 0 & 0 & 1
\end{array}\right) & , A_{2}=\left(\begin{array}{llll}
0 & 1 & 0 & 0 \\
1 & 0 & 0 & 0 \\
0 & 0 & 0 & 1 \\
0 & 0 & 0 & 0
\end{array}\right) \\
B_{1}=\left(\begin{array}{llll}
1 & 0 & 0 & 0 \\
0 & 1 & 0 & 0 \\
0 & 0 & 0 & 0 \\
0 & 0 & 0 & 0
\end{array}\right) & , B_{2}=\left(\begin{array}{llll}
1 & 0 & 0 & 0 \\
1 & 0 & 0 & 0 \\
0 & 0 & 1 & 0 \\
0 & 0 & 0 & 1
\end{array}\right) .
\end{array}
$$

The vectorial valued function $f(x)$ is defined by

$$
f(x)=\left(\begin{array}{c}
0 \\
0 \\
x^{2}-2 x \\
0
\end{array}\right)
$$

function $G(x, t)$ is

$$
G(x, t)=\left(\begin{array}{c}
-\cos (\pi x)\left(\cos (t)+2\left(t+\pi^{2} t^{2}+\pi^{2} \sin (t)\right)\right)+\frac{\sin (\pi x)}{\pi}\left(2 t\left(1+\pi^{2} t\right)\right) \\
-\cos (\pi x)\left(t\left(2+3 \pi^{2} t\right)+\pi^{2} \sin (t)\right)+\pi t^{2} \sin (\pi x) \\
e^{-t}(-1+x)^{2} x+\pi^{2} \cos (\pi x)\left(t^{2}+\sin (t)\right)-\pi t^{2} \sin (\pi x) \\
0
\end{array}\right),
$$


A method to solve non-homogeneous strongly coupled ...

and functions $P(t)$ and $Q(t)$ are defined by

$$
P(t)=\left(\begin{array}{c}
t^{2} \\
0 \\
0 \\
0
\end{array}\right), Q(t)=\left(\begin{array}{c}
0 \\
\sin (t) \\
0 \\
0
\end{array}\right)
$$

\section{We follow the Algorithm 1 STEP By STEP}

1. We will determine a vector valued function $v(x, t)$ fulfilling conditions (25). As any of the components of the vector valued function $G(x, t)$ are combinations of functions $\sin (\pi x)$ and $\cos (\pi x)$, we will look for $v(x, t)$ in the form given by (31). To do this, from (34) we obtain

$$
R_{1}(t)=\left(\begin{array}{c}
t^{2} / \pi \\
0 \\
0 \\
0
\end{array}\right), R_{2}(t)=\left(\begin{array}{c}
-t^{2}-\sin (t) \\
-t^{2} \\
0 \\
0
\end{array}\right)
$$

and thus determine the function $v(x, t)$ defined by

$$
v(x, t)=\left(\begin{array}{c}
-t^{2} \cos (\pi x)-\cos (\pi x) \sin (t)+\frac{t^{2} \sin (\pi x)}{\pi} \\
-t^{2} \cos (\pi x) \\
0 \\
0
\end{array}\right)
$$

where replacing in (35) one gets

$$
G_{1}(x, t)=\left(\begin{array}{c}
-\cos (\pi x)\left(\cos (t)+2\left(t+\pi^{2} t^{2}+\pi^{2} \sin (t)\right)\right)+\frac{2 t\left(1+\pi^{2} t\right) \sin (\pi x)}{\pi} \\
-\cos (\pi x)\left(t\left(2+3 \pi^{2} t\right)+\pi^{2} \sin (t)\right)+\pi t^{2} \sin (\pi x) \\
\pi\left(\pi \cos (\pi x)\left(t^{2}+\sin (t)\right)-t^{2} \sin (\pi x)\right) \\
0
\end{array}\right) .
$$

Thus, vector valued function $v(x, t)$ verifies trivially (25). 
2. From the definition of $v(x, t)$ we determine $\widetilde{G}(x, t)$ and $\widetilde{f(x)}$ :

$$
\begin{aligned}
& \widetilde{G}(x, t)=\left(\begin{array}{c}
0 \\
0 \\
(x-1)^{2} x e^{-t} \\
0
\end{array}\right), \\
& \tilde{f}(x)=f(x)=\left(\begin{array}{c}
0 \\
0 \\
x^{2}-2 x \\
0
\end{array}\right) .
\end{aligned}
$$

3. Using the algorithm given in Ref. [4] we can construct a solution $w(x, t)$ of problem (27)-(30) with these date. Observe that this problem is precisely the non-homogeneous problem with homogeneous conditions which was solved in the Example 3.2 of Ref. [4], whose exact solution is given by the series

$$
\begin{gathered}
w(x, t)= \\
\left(\sum_{n \geq 0}-\frac{32 e^{-\frac{1}{2}(\pi+2 n \pi)^{2} t} \sin \left(\frac{1}{2}(1+2 k) \pi x\right)}{\pi^{3}(2 k+1)^{3}}\right)\left(\begin{array}{l}
0 \\
0 \\
1 \\
0
\end{array}\right) \\
-\left(\sum_{n \geq 0} \frac{3072(-1)^{n} e^{-\frac{(2 n+1)^{2} \pi^{2} t}{2}}\left(e^{\frac{\left(-2+(2 n+1)^{2} \pi^{2}\right)^{t}}{2}}-1\right)\left((2 n+1)^{2} \pi^{2}-10\right) \sin \left(\frac{(2 n+1) \pi x}{2}\right)}{(2 n+1)^{6} \pi^{6}\left(-2+(2 n+1)^{2} \pi^{2}\right)}\right)\left(\begin{array}{l}
0 \\
0 \\
1 \\
0
\end{array}\right) .
\end{gathered}
$$

4. The solution of problem (20)-(23) is given by $u(x, t)=w(x, t)+v(x, t)$, i.e., by the expression:

$$
\begin{aligned}
& u(x, t)= \\
& \left(\sum_{n \geq 0}-\frac{32 e^{-\frac{1}{2}(\pi+2 n \pi)^{2} t} \sin \left(\frac{1}{2}(1+2 k) \pi x\right)}{\pi^{3}(2 k+1)^{3}}\right)\left(\begin{array}{l}
0 \\
0 \\
1 \\
0
\end{array}\right) \\
& -\left(\sum_{n \geq 0} \frac{3072(-1)^{n} e^{-\frac{(2 n+1)^{2} \pi^{2} t}{2}}\left(e^{\frac{\left(-2+(2 n+1)^{2} \pi^{2}\right) t}{2}}-1\right)\left((2 n+1)^{2} \pi^{2}-10\right) \sin \left(\frac{(2 n+1) \pi x}{2}\right)}{(2 n+1)^{6} \pi^{6}\left(-2+(2 n+1)^{2} \pi^{2}\right)}\right)\left(\begin{array}{l}
0 \\
0 \\
1 \\
0
\end{array}\right) \\
& +\left(\begin{array}{c}
-t^{2} \cos (\pi x)-\cos (\pi x) \sin (t)+\frac{t^{2} \sin (\pi x)}{\pi} \\
-t^{2} \cos (\pi x) \\
0 \\
0
\end{array}\right) .
\end{aligned}
$$


Example 3.2 We consider problem (20)-(23). Suppose that $G(x, t)$ is a polynomial in $x$, with coefficients are functions of the variable $t$. Thus, we look for a vector valued function $v(x, t)$ which is also a polynomial in $x$ (cubic, for example), whose coefficients are functions of the variable $t$, in the form

$$
v(x, t)=R_{3}(t) x^{3}+R_{2}(t) x^{2}+R_{1}(t) x+R_{0}(t),
$$

where functions $R_{i}(t) \in \mathcal{C}^{1}[0,+\infty), i=0,1,2,3$ must be determinate. This function (41) satisfy the boundary conditions (25), i.e.

$$
\left.\begin{array}{rl}
A_{1} R_{0}(t)+B_{1} R_{1}(t) & =P(t) \\
A_{2}\left(R_{3}(t)+R_{2}(t)+R_{1}(t)+R_{0}(t)\right)+B_{2}\left(3 R_{3}(t)+2 R_{2}(t)+R_{1}(t)\right) & =Q(t) .
\end{array}\right\}
$$

we can write the above system in matrix form:

$$
\left(\begin{array}{cccc}
A_{1} & B_{1} & 0 & 0 \\
A_{2} & A_{2}+B_{2} & A_{2}+2 B_{2} & A_{2}+3 B_{2}
\end{array}\right)\left(\begin{array}{l}
R_{0}(t) \\
R_{1}(t) \\
R_{2}(t) \\
R_{3}(t)
\end{array}\right)=\left(\begin{array}{c}
P(t) \\
Q(t)
\end{array}\right)
$$

Taking block matrices

$$
\hat{A}=\left(\begin{array}{rrrr}
I & 0 & 0 & 0 \\
0 & I & 0 & 0 \\
0 & -3 I & I & 0 \\
0 & 2 I & 0 & I
\end{array}\right), \hat{B}=\left(\begin{array}{rrrr}
I & 0 & 0 & 0 \\
0 & I & 0 & 0 \\
0 & 3 I & I & 0 \\
0 & -2 I & 0 & I
\end{array}\right)
$$

which trivially satisfy that $\hat{A} \hat{B}=I$, (42) can be writen in the form

$$
\left(\begin{array}{cccc}
A_{1} & B_{1} & 0 & 0 \\
A_{2} & A_{2}+B_{2} & A_{2}+2 B_{2} & A_{2}+3 B_{2}
\end{array}\right) \hat{A} \hat{B}\left(\begin{array}{c}
R_{0}(t) \\
R_{1}(t) \\
R_{2}(t) \\
R_{3}(t)
\end{array}\right)=\left(\begin{array}{c}
P(t) \\
Q(t)
\end{array}\right)
$$

thus

$$
\left(\begin{array}{cccc}
A_{1} & B_{1} & 0 & 0 \\
A_{2} & B_{2} & A_{2}+2 B_{2} & A_{2}+3 B_{2}
\end{array}\right)\left(\begin{array}{c}
R_{0}(t) \\
R_{1}(t) \\
3 R_{1}(t)+R_{2}(t) \\
R_{3}(t)-2 R_{1}(t)
\end{array}\right)=\left(\begin{array}{c}
P(t) \\
Q(t)
\end{array}\right)
$$

We can rewrite (43) in the form 
$\left(\begin{array}{ll}A_{1} & B_{1} \\ A_{2} & B_{2}\end{array}\right)\left(\begin{array}{l}R_{0}(t) \\ R_{1}(t)\end{array}\right)+\left(\begin{array}{cc}0 & 0 \\ A_{2}+2 B_{2} & A_{2}+3 B_{2}\end{array}\right)\left(\begin{array}{c}R_{2}(t)+3 R_{1}(t) \\ R_{3}(t)-2 R_{1}(t)\end{array}\right)=\left(\begin{array}{c}P(t) \\ Q(t)\end{array}\right)$

If we impose the condition:

$$
\left(\begin{array}{cc}
0 & 0 \\
A_{2}+2 B_{2} & A_{2}+3 B_{2}
\end{array}\right)\left(\begin{array}{l}
R_{2}(t)+3 R_{1}(t) \\
R_{3}(t)-2 R_{1}(t)
\end{array}\right)=\left(\begin{array}{l}
0 \\
0
\end{array}\right)
$$

or equivalently:

$$
\left.\begin{array}{l}
R_{2}(t)=-3 R_{1}(t) \\
R_{3}(t)=2 R_{1}(t)
\end{array}\right\}
$$

from (44) we have the matrix block system

$$
\left(\begin{array}{ll}
A_{1} & B_{1} \\
A_{2} & B_{2}
\end{array}\right)\left(\begin{array}{l}
R_{0}(t) \\
R_{1}(t)
\end{array}\right)=\left(\begin{array}{c}
P(t) \\
Q(t)
\end{array}\right)
$$

Taking into account (6), system (46) have an unique solution. Thus, we have shown that we can determine a vector valued function $v(x, t)$ satisfying conditions (25) and defined by expression (31). Thus, we have now that

$$
\begin{aligned}
G_{1}(x, t) & =v_{t}(x, t)-A v_{x x}(x, t) \\
& =R_{3}^{\prime}(t) x^{3}+R_{2}^{\prime}(t) x^{2}+R_{1}^{\prime}(t) x+R_{0}^{\prime}(t)-A\left(6 R_{3}(t) x+2 R_{2}(t) \gamma\right.
\end{aligned}
$$

and we can apply Theorem 2.1. We will consider a concrete numerical example. Consider problem (20)-(23) where matrix $A \in \mathbb{C}^{4 \times 4}$ is given by

$$
A=\left(\begin{array}{rrrr}
2 & 0 & 0 & -1 \\
1 & 2 & 1 & -2 \\
-1 & 0 & 2 & 1 \\
0 & 0 & 0 & 1
\end{array}\right)
$$

and the matrices $A_{i}, B_{i}, i \in\{1,2\}$ given by (37). Also, the vectorial valued functions $f(x)$ and $G(x, t)$ will be defined respectively as

$$
f(x)=\left(\begin{array}{c}
0 \\
x^{2}-1 \\
0 \\
0
\end{array}\right)
$$

and 


$$
G(x, t)=\left(\begin{array}{c}
\cos (t)-2 t+12 t^{2}+2 t x-24 t^{2} x-6 t x^{2}+4 t x^{3} \\
-2 t+6 t^{2}-12 t^{2} x+e^{-t} x^{3}-2 e^{-t} x^{4}+e^{-t} x^{5} \\
-6 t^{2}+12 t^{2} x \\
0
\end{array}\right)
$$

and functions $P(t)$ and $Q(t)$ defined by (40).

\section{We follow the Algorithm 1 STEP BY STEP}

1. We will determine a vector valued function $v(x, t)$ fulfilling conditions (25). As any of the components of the vector valued function $G(x, t)$ are polynomials in the variable $x$, with coefficients are functions of the variable $t$, we will look for $v(x, t)$ in the form given by (41). To do this, from (46) we obtain

$$
R_{0}(t)=\left(\begin{array}{c}
\sin (t)-t^{2} \\
-t^{2} \\
0 \\
0
\end{array}\right), R_{1}(t)=\left(\begin{array}{l}
t^{2} \\
0 \\
0 \\
0
\end{array}\right)
$$

and from (45) we obtain

$$
R_{2}(t)=\left(\begin{array}{c}
-3 t^{2} \\
0 \\
0 \\
0
\end{array}\right), R_{3}(t)=\left(\begin{array}{c}
2 t^{2} \\
0 \\
0 \\
0
\end{array}\right)
$$

and therefore we have the function

$$
v(x, t)=\left(\begin{array}{c}
-t^{2}+t^{2} x-3 t^{2} x^{2}+2 t^{2} x^{3}+\sin (t) \\
-t^{2} \\
0 \\
0
\end{array}\right)
$$

From (47) one gets

$$
G_{1}(x, t)=\left(\begin{array}{c}
-2 t+12 t^{2}+2 t x-24 t^{2} x-6 t x^{2}+4 t x^{3}+\cos (t) \\
-2 t+6 t^{2}-12 t^{2} x \\
-6 t^{2}+12 t^{2} x \\
0
\end{array}\right)
$$

Thus, vector valued function $v(x, t)$ verifies trivially (25). 
2. From the definition of $v(x, t)$ we determine $\widetilde{G}(x, t)$ and $\widetilde{f(x)}$ :

$$
\begin{gathered}
\widetilde{G}(x, t)=\left(\begin{array}{c}
0 \\
(x-1)^{2} x^{3} e^{-t} \\
0 \\
0
\end{array}\right), \\
\widetilde{f}(x)=f(x)=\left(\begin{array}{c}
0 \\
x^{2}-1 \\
0 \\
0
\end{array}\right) .
\end{gathered}
$$

3. Using the algorithm given in Ref. [4] we can construct a solution $w(x, t)$ of problem (27)-(30) with these date. Observe that this problem is precisely the non-homogeneous problem with homogeneous conditions which was solved in the Example 3.1 of Ref. [4], whose exact solution is given by the series

$$
\begin{gathered}
w(x, t)= \\
\left(\sum_{n \geq 0}-\frac{32(-1)^{n} e^{-\frac{1}{2}(\pi+2 n \pi)^{2} t} \cos \left(\frac{1}{2}(2 n+1) \pi x\right)}{\pi^{3}(2 n+1)^{3}}\right)\left(\begin{array}{l}
0 \\
1 \\
0 \\
0
\end{array}\right) \\
+\left(\sum_{n \geq 0}-\frac{64 e^{-\frac{(2 n+1)^{2} \pi^{2} t}{2}}\left(e^{\frac{\left(-2+(2 n+1)^{2} \pi^{2}\right) t}{2}}-1\right) \mathcal{A}(n) \cos \left(\frac{(2 n+1) \pi x}{2}\right)}{(2 n+1)^{6} \pi^{6}\left(-2+(2 n+1)^{2} \pi^{2}\right)}\right)\left(\begin{array}{l}
0 \\
1 \\
0 \\
0
\end{array}\right)
\end{gathered}
$$

where

$$
\mathcal{A}(n)=\left(480+(2 n+1) \pi\left(-144(-1)^{n}+(2 n+1) \pi\left((-1)^{n}(2 n+1) \pi-6\right)\right)\right) .
$$

4. The solution of problem (20)-(23) is given by $u(x, t)=w(x, t)+v(x, t)$, i.e., by the expression:

$$
u(x, t)=
$$




$$
\begin{aligned}
& \left(\sum_{n \geq 0}-\frac{32(-1)^{n} e^{-\frac{1}{2}(\pi+2 n \pi)^{2} t} \cos \left(\frac{1}{2}(2 n+1) \pi x\right)}{\pi^{3}(2 n+1)^{3}}\right)\left(\begin{array}{l}
0 \\
1 \\
0 \\
0
\end{array}\right) \\
& +\left(\sum_{n \geq 0}-\frac{64 e^{-\frac{(2 n+1)^{2} \pi^{2} t}{2}}\left(e^{\frac{\left(-2+(2 n+1)^{2} \pi^{2}\right) t}{2}}-1\right) \mathcal{A}(n) \cos \left(\frac{(2 n+1) \pi x}{2}\right)}{(2 n+1)^{6} \pi^{6}\left(-2+(2 n+1)^{2} \pi^{2}\right)}\right)\left(\begin{array}{l}
0 \\
1 \\
0 \\
0
\end{array}\right) \\
& +\left(\begin{array}{c}
-t^{2}+t^{2} x-3 t^{2} x^{2}+2 t^{2} x^{3}+\sin (t) \\
-t^{2} \\
0 \\
0
\end{array}\right)
\end{aligned}
$$

where

$$
\mathcal{A}(n)=\left(480+(2 n+1) \pi\left(-144(-1)^{n}+(2 n+1) \pi\left((-1)^{n}(2 n+1) \pi-6\right)\right)\right) .
$$

\section{Conclusion}

In this paper a method to solve non-homogeneous problem with non-homogeneous conditions of the type (20)-(23) in terms of the solution of a non-homogeneous with homogeneous conditions problem (16)-(19) with appropriate parameters, is developed. The computational process is outlined in Algorithm 1. The choose of the appropriate function $v(x, t)$ is illustrated in the examples 3.1 and 3.2 .

\section{References}

[1] S. L. Campbell, C. D. Meyer jr,. Generalized Inverses of Linear Transformations, Pitman, London, 1979. http://dx.doi.org/10.1002/zamm.19810610820

[2] V. Soler, E. Defez, M. V. Ferrer, J. Camacho, On exact series solution of strongly coupled mixed parabolic problems, Abstract and Applied Analysis, Article ID 524514 (2013). http://dx.doi.org/10.1155/2013/524514

[3] V. Soler, E. Defez, J. A. Verdoy, On exact series solution for strongly coupled mixed parabolic boundary value problems, Abstract and Applied Analysis, Article ID 759427 (2014). http://dx.doi.org/10.1155/2014/759427 
[4] V. Soler, E. Defez, R. Capilla, J. A. Verdoy, On the exact series solution for non-homogeneous strongly coupled mixed parabolic boundary value problems, Abstract and Applied Analysis, Article ID 826860 (2014). http://dx.doi.org/10.1155/2014/826860

Received: July 16, 2015; Published: July 29, 2015 